\title{
Efectos de la nicotina en el grosor coroideo mediante tomografía de coherencia óptica con imagen de profundidad mejorada
}

\section{Effects of nicotine on choroidal thickness by enhanced depth imaging optical coherence tomography}

\author{
Yolanda Villalpando-Gómez ${ }^{1 *}$, Itzel Espinosa-Soto ${ }^{1}$, Juan A. Ramírez-Estudillo ${ }^{1}$ y \\ Atzin Robles-Contreras ${ }^{2}$ \\ ${ }^{1}$ Departamento de Retina y Vitreo; ${ }^{2}$ Centro de Investigación Biomédica. Hospital Nuestra Señora de la Luz, Ciudad de México, México
}

\begin{abstract}
Resumen
Introducción: El tabaquismo y la nicotina son factores de riesgo conocidos para diversas enfermedades cardiovasculares, por lo que es de esperar que estos se asocien a cambios vasculares en diversas partes del organismo, incluyendo la vasculatura coroidea. Objetivo: Evaluar los efectos que tiene la nicotina en el grosor coroideo en pacientes sanos medido mediante la tomografía de coherencia óptica con imagen de profundidad mejorada (EDI-OCT). Material y métodos: Estudio de casos y controles, en el cual se midió el grosor coroideo a sujetos sanos tras consumir una goma de mascar con $4 \mathrm{mg}$ de nicotina y se comparó con un grupo control al cual se le administró una goma de mascar sin nicotina. Para la estadística descriptiva se utilizaron frecuencias, medias y desviación estándar. Para la estadística inferencial se realizó un análisis de varianza de un factor para la comparación intergrupo. Resultados: Se incluyeron 64 ojos. En la comparación del grosor coroideo en el grupo control en el momento basal, a la hora y a las dos horas, no se observó una diferencia significativa ( $p=0.29$ y $p=0.13$, respectivamente). Por otra parte, al realizar la comparación del grupo con nicotina, no observamos una diferencia estadísticamente significativa entre el grosor coroideo basal, a la hora y las dos horas ( $p=0.57$ y $p=1.43$, respectivamente). Conclusión: En nuestro estudio no observamos que la goma de mascar con nicotina generara cambios transitorios en el grosor coroideo. Estos cambios podrían estar relacionados con el consumo crónico de nicotina y con el daño consiguiente generado por este consumo.
\end{abstract}

Palabras clave: Grosor coroideo. Nicotina. Tomografía de coherencia óptica.

\section{Abstract}

Background: Smoking and nicotine are known risk factors for cardiovascular diseases, so it is expected that these are associated with vascular changes in the organism, including the choroidal vasculature. Purpose: To evaluate the effects of nicotine on choroidal thickness in healthy patients by enhanced depth imaging optical coherence tomography (EDI-OCT). Methods: Case-control study, in which choroidal thickness was measured in healthy subjects after consuming a chewing gum with $4 \mathrm{mg}$ nicotine and compared with a control group given a nicotine-free chewing gum. For descriptive statistics

Correspondencia:

*Yolanda Villalpando-Gómez

Ezequiel Montes, 135

Col. Tabacalera, Del. Cuauhtémoc Fecha de recepción: 23-02-2017

Fecha de aceptación: 21-06-2017

Disponible en internet: 14-05-2018

Ciudad de México, México

DOI: 10.24875/RMO.M18000020

Rev Mex Oftalmol. 2018;92(3):123-128

E-mail: yolavg@me.com

gía. Publicado por Permanyer México. Este es un artículo Open Access bajo la licencia CC BY-NC-ND

(http://creativecommons.org/licenses/by-nc-nd/4.0/). 
frequencies, means and standard deviation were used. For inferential statistics, one-way analysis of variance was used for intergroup comparison. Results: Sixty four eyes were included. In the comparison of choroidal thickness in the control group at baseline, at $1 \mathrm{hr}$ and $2 \mathrm{hrs}$, no significant difference was observed ( $p=0.29, p=0.13$, respectively). Otherwise, when we performed the comparison of the group with nicotine, we did not observe a statistical significance between basal choroidal thickness, at $1 \mathrm{hr}$ and 2 rhrs ( $p=0.57, p=1.43$, respectively). Conclusion: In our study we did not observe that the chewing gum with nicotine generated transient changes in the choroidal thickness, these changes could be related to the chronic consumption of nicotine and with the consequent damage generated by it.

Key words: Choroidal thickness. Nicotine. Optical coherence tomography.

\section{Introducción}

La coroides es la estructura vascular con mayor flujo sanguíneo dentro del organismo. Es una estructura compleja, encargada de suministrar oxígeno a las capas externas de la retina, y su estudio ha sido de gran interés a lo largo de los años ${ }^{1}$.

La tomografía de coherencia óptica (OCT) es un estudio no invasivo que se ha utilizado para visualizar la morfología de las capas de la retina; sin embargo, la anatomía coriodea se visualiza poco debido a la baja penetración de la señal a través del epitelio pigmentado de la retina ${ }^{2,3}$.

Por medio de la OCT, a través de la imagen de profundidad mejorada (EDI-OCT), que representa una modalidad de obtención de imagen basada en un software que utiliza las características del dominio espectral-OCT (SD-OCT), como son el número de escaneos (más de 100) y el rastreo ocular (eye tracking), al disminuir la distancia entre el dispositivo y el ojo a explorar, se obtiene una imagen en espejo invertida, lo que permite una mejor visualizacion de la coroides y la unión coroido-escleral ${ }^{4}$.

Se ha observado, mediante la medición del grosor coroideo a través de la EDI-OCT, que dicha estructura tiene un papel muy importante en la fisiopatología de enfermedades como la coriorretinopatía serosa central $^{5}$, la vasculopatía coroidea polipoidal ${ }^{6,7}$, el síndrome de Vogt-Koyanagi-Harada ${ }^{8-10} \mathrm{o}$ la degeneración macular relacionada con la edad ${ }^{11-14}$.

El tabaquismo crónico es considerado un factor de riesgo para diversas enfermedades vasculares oculares, y su fisiopatología no es del todo conocida; $\sin$ embargo, se ha observado que este da como resultado un aumento de la resistencia vascular coroidea ${ }^{15,16}$.

Se ha reportado una disminución en el grosor coroideo en pacientes fumadores, la cual se mantiene hasta tres horas después de fumar ${ }^{17}$. Se ha descrito también una disminución del grosor coroideo en pacientes fumadores sanos; sin embargo, se ha observado que este efecto es transitorio y que vuelve a la normalidad una hora después de haber fumado un cigarrillo. No se ha encontrado una diferencia estadísticamente significativa en la OCT basal al compararla con los controles $^{18}$.

Sanchez, et al., en un estudio realizado en 26 personas, observaron una disminución del grosor coroideo subfoveal estadísticamente significativo en pacientes fumadores al compararlos con no fumadores, que se relaciona con la disminución del flujo coroideo ocasionado por la vasoconstricción secundaria a la nicotina del tabaquismo ${ }^{19}$.

En nuestro estudio analizamos el cambio causado únicamente por la nicotina en el grosor coroideo y no de todos los componentes químicos del cigarrillo como tal. Por otra parte, existen pocos reportes sobre cambios en el grosor coroideo secundarios al consumo de nicotina en población mexicana.

\section{Material y métodos}

Se trata de un estudio de casos y controles en el cual se incluyeron personas sanas que decidieron participar en el mismo, firmaron un consentimiento informado y tenían que ser mayores de 18 años, de género indistinto y sin patologías oftalmológicas o sistémicas adyacentes (no se excluyeron fumadores). Se excluyeron pacientes de sexo femenino en gestación. Se tuvo un grupo control al cual se le administró una goma de mascar sin nicotina y un grupo de casos que ingirió un chicle con $4 \mathrm{mg}$ de nicotina. Este trabajo fue aprobado por el comité local de ética en investigación de acuerdo al riesgo de la Ley General de Salud y la Declaración de Helsinki.

Se realizó la toma de EDI-OCT bajo dilatación entre las 9 y las $11 \mathrm{~h}$ de la mañana para la toma basal en ambos grupos, posteriormente a cada individuo se le dio una goma de mascar de $4 \mathrm{mg}$ de nicotina o una goma común de caramelo. Fueron asignados de manera aleatoria por medio de una urna al grupo de casos 0 al grupo control. Ni el paciente ni el investigador principal conocían a cuál de los grupos pertenecía 
cada individuo. Se solicitó que las gomas de mascar fueran masticadas durante al menos 10 minutos, y el estudio se repitió a la hora y a las dos horas. La medición en micras se realizó a nivel subfoveal desde la cara externa de la línea hiperrefléctica correspondiente al complejo epitelio pigmentado/membrana de Bruch hasta la cara interna de la línea hiperrefléctica correspondiente a la unión coroido-escleral (grosor vascular coroideo según la clasificación de Yiu, et al.).

Para la obtención de imágenes se utilizó el dispositivo OCT de dominio espectral Heidelberg mediante la imagen de profundidad mejorada y fueron evaluadas por un solo explorador.

Las variables a considerar para el análisis fueron: edad del paciente, grosor coroideo basal comparado con el grosor coroideo a la hora, a las dos horas y a las tres horas.

Para el análisis estadístico se utilizó el programa GraphPad Prism (versión 5.00) y la estadística descriptiva se realizó con promedios, frecuencias, desviación estándar, media y rangos. Para la estadística inferencial se establecieron diferencias entre grupos utilizando la prueba t-Student y diferencias intergrupos realizando un análisis de varianza de un factor.

\section{Consideraciones éticas}

Se trata de un estudio intervencional que analizó imágenes obtenidas mediante un procedimiento no invasivo. A los sujetos del grupo de los casos se les administró una goma de $4 \mathrm{mg}$ de nicotina, la cual se ha utilizado en estudios similares. Los niveles de nicotina alcanzados con la goma de mascar son inferiores a los que ofrece un cigarrillo, además de que el riesgo de adicción es nulo. Este trabajo fue aprobado por el comité local de ética en investigación de acuerdo al riesgo de la Ley General de Salud y la Declaración de Helsinki. Se firmó un consentimiento informado.

\section{Riesgos del estudio para los participantes}

Los riesgos asociados al consumo de la goma de mascar con nicotina descritos fueron efectos adversos leves, como ardor de garganta, cefalea o náuseas. El uso de estas gomas no se recomienda en mujeres embarazadas o en periodos de lactancia, aunque estas las han utilizado por tener un efecto menos nocivo que los cigarrillos; sin embargo, para mayor seguridad del protocolo, este grupo de sujetos se excluyeron del estudio.
Tabla 1. Características generales

\begin{tabular}{|l|l|c|}
\hline & & $\begin{array}{c}\text { Pacientes } \\
\mathrm{n}=32\end{array}$ \\
\hline Edad & & $28.09 \pm 2(25-34)$ \\
\hline Sexo $(\%)$ & $\begin{array}{l}\text { Memenino } \\
\text { Masculino }\end{array}$ & $11(34.4)$ \\
\hline Ocupación (\%) & Estudiantes & $32(65.6)$ \\
\hline APP (\%) & Negados & $32(100)$ \\
\hline APNP (\%) & Tabaquismo & $10(31.3)$ \\
\hline
\end{tabular}

APNP: antecedentes personales no patológicos; APP: antecedentes personales patológicos.

Tabla 2. Grosor coroideo subfoveal

\begin{tabular}{|l|l|l|}
\hline & Sin nicotina & Con nicotina \\
\hline Basal $^{*}$ & $324.1 \pm 72.25$ & $355.0 \pm 94.85$ \\
\hline 1 hora* $^{*}$ & $323.7 \pm 71.45$ & $355.2 \pm 96.34$ \\
\hline 2 horas $^{*}$ & $323.0 \pm 72.93$ & $352.8 \pm 94.70$ \\
\hline
\end{tabular}

*Media.

\section{Resultados}

Se incluyeron 64 ojos de 32 pacientes, donde observamos dentro de las características generales que la edad media fue de $28 \pm 2$ años, predominaban los pacientes de sexo masculino en un $65.6 \%$ y eran estudiantes en su totalidad (Tabla 1).

Dentro de los antecedentes personales no patológicos, el $31 \%$ de los pacientes referían tabaquismo positivo y sin antecedentes personales patológicos de importancia (Tabla 1).

Del total de pacientes, el $53.1 \%$ correspondieron al grupo control (goma de mascar sin nicotina) y el $46.8 \%$ al grupo de goma de mascar con nicotina.

La media observada del grosor coroideo subfoveal basal en el grupo control fue de $324.1 \mu \mathrm{m}$, a la hora, de $323.7 \mu \mathrm{m}$, y a las dos horas, de $323.0 \mu \mathrm{m}$, correspondiendo en el grupo de goma de mascar con nicotina en la toma basal a $355.0 \mu \mathrm{m}$, a la hora a $355.2 \mu \mathrm{m}$ y a las dos horas a $352.8 \mu \mathrm{m}$ (Tabla 2).

Al realizar la comparación entre grupos, dentro del grupo de goma de mascar sin nicotina, no se observó una diferencia estadísticamente significativa entre el grosor coroideo subfoveal basal, a la hora y a las dos horas ( $p=0.29$ y $p=0.13$, respectivamente). Por otra parte, al realizar la correlación en el grupo de goma de 
mascar con nicotina entre el grosor coroideo basal, la primera hora y la segunda hora tampoco existió una diferencia estadísticamente significativa $(p=0.57 \mathrm{y}$ $p=1.43$, respectivamente) (Fig. 1).

Se realizó una comparación entre el grupo de goma de mascar con nicotina basal vs. sin nicotina basal, con nicotina a la hora vs. sin nicotina a la hora y con nicotina a las dos horas vs. sin nicotina a las dos horas para observar si existía alguna diferencia; sin embargo, al igual que en las anteriores correlaciones, esta no fue significativa ( $p=0.13, p=0.10$ y $p=0.14$, respectivamente).

Por último, se realizó una comparación entre fumadores y no fumadores entre la medición basal, a la hora y a las dos horas, y no se observó una diferencia estadísticamente significativa del grosor coroideo subfoveal (Fig. 2).

\section{Discusión}

Al realizar el análisis de nuestros resultados observamos que no existió una diferencia estadísticamente significativa del grosor coroideo basal entre el grupo control y el grupo de estudio. De igual forma, al realizar una comparación intergrupo toma basal vs. una hora, basal vs. dos horas, así como también una hora vs. dos horas, en ambos grupos no se encontró una diferencia significativa. Por otra parte, al realizar la comparación entre grupos a la hora en el grupo con nicotina vs. a la hora en el grupo sin nicotina, así como a las dos horas entre ambos grupos, basal vs. una hora, basal vs. dos horas y una hora vs. dos horas, no se observó una diferencia estadísticamente significativa.

Sizmaz, et al., en su estudio realizado en personas fumadoras (grupo de estudio) y no fumadoras (grupo control), donde los participantes del grupo de estudio se fumaron un cirgarrillo, realizaron una medición del grosor coroideo a ambos grupos en el momento basal, a la hora y a las tres horas, reportando, a diferencia de nuestro estudio, una disminución del grosor coroideo estadísticamente significativa a la hora y a las tres horas al compararlo con la medición basal en el grupo de estudio ${ }^{17}$. Dicha diferencia podría ser explicada debido a la mayor concentración plasmática de nicotina alcanzada al fumarse un cigarrillo vs. la ingesta oral.

Por otra parte, Ulas, et al., al evaluar los efectos producidos por el tabaquismo sobre el grosor coroideo subfoveal, observaron un aumento del mismo en los primeros 5 minutos posteriores a fumarse un cigarrillo, y un regreso al grosor coroideo basal a la hora. Dicho cambio puede hacernos pensar en un comportamiento

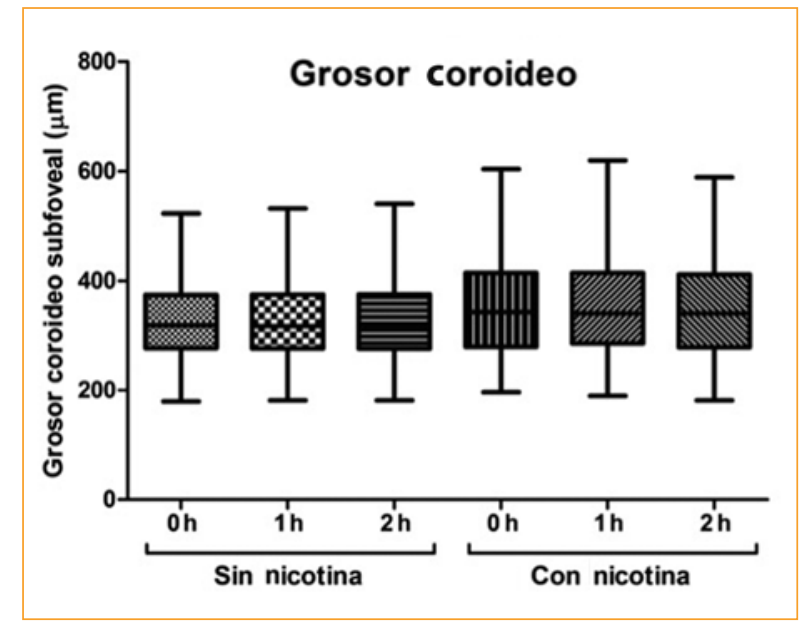

Figura 1. Comparación entre grupos de grosor coroideo.

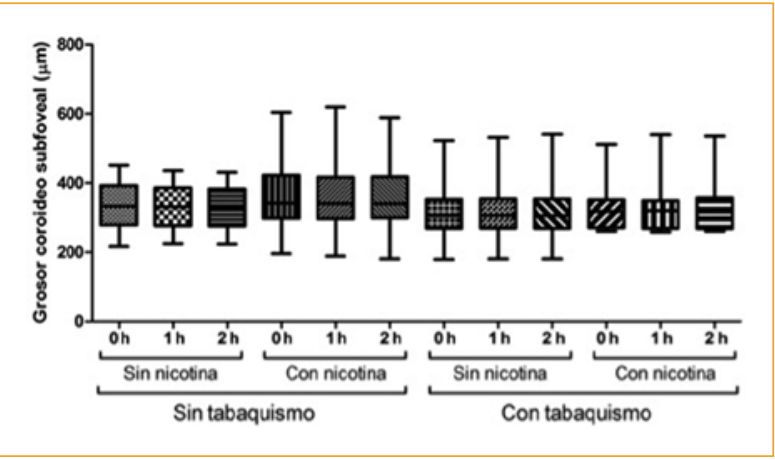

Figura 2. Comparación de grosor coroideo entre fumadores y no fumadores.

mucho más dinámico de la coroides del que creemos, por lo que sería de gran utilidad realizar mediciones más tempranas ${ }^{18}$.

Moschos, et al., en un estudio publicado durante el 2016, en el cual incluyeron pacientes fumadores de 25 años de evolución y al menos una cajetilla diaria durante al menos 1 año vs. controles, reportaron una disminución significativa del grosor coroideo. Esta diferencia observada con nuestro estudio podría estar relacionada, como mencionan en sus conclusiones, a los cambios causados por el tabaquismo crónico, ya que se relaciona con una disfunción endotelial y reactividad coroidea inducida por el tabaquismo 20 .

Tayyab, et al., al igual que lo observado en nuestro estudio, en un grupo de 80 pacientes en el cual incluyeron fumadores y no fumadores, observaron que no 
existía una diferencia estadísticamente significativa del grosor coroideo entre ambos grupos ${ }^{21}$.

Benowitz, et al. reportan que la goma de mascar con nicotina contiene la mitad de la cantidad de nicotina que contiene un cigarrillo y que el mayor nivel de nicotina en sangre se alcanza a los 30 minutos, siendo este aumento gradual, persistiendo, y disminuyendo lentamente hasta después de las dos horas, motivo por el cual, en nuestro estudio realizamos la segunda toma a la hora, momento en que aún se mantienen los niveles de nicotina plasmáticos, y la última, a las tres horas, momento en el cual dichos niveles han disminuido ${ }^{22}$.

En contraste con nuestro estudio, Zengin, et al., en un estudio realizado en 32 pacientes, en el cual investigaron el efecto de la nicotina en el grosor coroideo utilizando de igual forma goma de mascar con nicotina de $4 \mathrm{mg}$, reportaron un adelgazamiento del grosor coroideo. Dicha diferencia con nuestro estudio podría estar relacionada con el tiempo de uso de la goma de mascar con nicotina, ya que en nuestro estudio se mascó durante 10 minutos y en el estudio antes mencionado durante una hora, ello podría explicar la existencia de una retención de nicotina en la goma de mascar $^{23}$.

Nuestros hallazgos, al no encontrar diferencias del grosor coroideo entre el grupo de goma de mascar con nicotina y el grupo control, a pesar de haber sido medidos a la hora y a las dos horas posteriores, tiempo en el cual se alcanzan altos niveles de nicotina en sangre, el cual se mantiene, y posteriormente disminuye después de las dos horas de su administración, nos muestran que en nuestro estudio la goma de mascar con nicotina no tuvo efectos transitorios sobre el grosor coroideo subfoveal.

Por otra parte, podríamos decir que los cambios descritos en la vasculatura acerca del daño endotelial y el aumento de la resistencia vascular podrían estar relacionados con el daño crónico secundario al tiempo de exposición y el número de cigarrillos fumados al día (índice tabáquico).

Por lo que concluimos que en nuestro estudio no se observaron cambios transitorios al grosor coroideo con la goma de mascar de $4 \mathrm{mg}$ de nicotina y que los cambios reportados en la vasculatura coroidea asociados a la nicotina podrían estar relacionados con el consumo crónico de la misma. Sería de gran utilidad, en estudios futuros, medir los niveles plasmáticos de nicotina para conocer los niveles de absorción y el pico máximo de nicotina, y así, realizar una correlación más específica.
Nuestro estudio cuenta con algunas limitaciones, como el número reducido de pacientes, la medición únicamente subfoveal y la homogeneidad de la muestra.

\section{Responsabilidades éticas}

Protección de personas y animales. Los autores declaran que para esta investigación no se han realizado experimentos en seres humanos ni en animales.

Confidencialidad de los datos. Los autores declaran que han seguido los protocolos de su centro de trabajo sobre la publicación de datos de pacientes.

Derecho a la privacidad y consentimiento informado. Los autores han obtenido el consentimiento informado de los pacientes y/o sujetos referidos en el artículo. Este documento obra en poder del autor de correspondencia.

\section{Financiamiento}

No se obtuvo ningún financiamiento.

\section{Conflicto de intereses}

No existe ningún conflicto de interés de los investigadores en este estudio.

\section{Bibliografía}

1. Yiu G, Pecen P, Sarin N, Chiu SJ, Farsiu S, Mruthyunjaya P, et al. Characterization of the Choroid-Scleral Junction and Suprachoroidal Layer in Healthy Individuals on Enhanced-Depth Imaging Optical Coherence Tomography. JAMA Ophthalmol. 2014;132(2):174-81.

2. Fujimoto JG. Optical coherence tomography. CR Acad Sci Paris. 2001;2(8):1099-111.

3. Lin P, Mettu PS, Pomerleau DL, Chiu SJ, Maldonado R, Stinnett S, et al. Image inversion spectral-domain optical coherence tomography optimizes choroidal thickness and detail through improved contrast. Invest Ophthalmol Vis Sci. 2012;53(4):1874-82.

4. Spaide R, Koizumi H, Pozonni M. Enhanced Depth Imaging Spectral-Domain Optical Coherence Tomography. Am J Ophthalmol. 2008;146:496-500.

5. Maruko I, lida T, Sugano Y, Ojima A, Ogasawara M, Spaide RF. Subfoveal choroidal thickness after treatment of central serous chorioretinopathy. Ophthalmology. 2010;117(9):1792-9.

6. Chung SE, Kang SW, Lee JH, Kim YT. Choroidal thickness in polypoidal choroidal vasculopathy and exudative age-relatedmacular degeneration. Ophthalmology. 2011;118(5):840-5.

7. Jirarattanasopa $P$, Ooto $S$, Nakata I, Tsujikawa A, Yamashiro K, Oishi A et al. Choroidal thickness, vascular hyperpermeability, and complement factor $\mathrm{H}$ in age-relatedmacular degeneration and polypoidal choroidal vasculopathy. Invest Ophthalmol Vis Sci. 2012;53(7):3663-72.

8. Nakayama M, Keino H, Okada AA, Watanabe T, Taki W, Inoue M, et al Enhanced depth imaging optical coherence tomography of the choroid in Vogt-Koyanagi-Harada disease. Retina. 2012;32(10):2061-9.

9. Maruko I, lida T, Sugano Y, Oyamada H, Sekiryu T, Fujiwara T, et al. Subfoveal choroidal thickness after treatment of Vogt-Koyanagi-Harada disease. Retina. 2011;31(3):510-7.

10. Da Silva FT, Sakata VM, Nakashima A, Hirata CE, Olivalves E, Takahashi WY, et al. Enhanced depth imaging optical coherence tomography in long-standing Vogt-Koyanagi- Harada disease. $\mathrm{Br} \mathrm{J}$ Ophthalmol. 2013;97(1):70-4.

11. Switzer DW Jr, Mendonça LS, Saito M, Zweifel SA, Spaide RF. Segregation of ophthalmoscopic characteristics according to choroidal thickness in patients with early age-related macular degeneration. Retina. 2012;32(7):1265-71. 
Rev Mex Oftalmol. 2018;92

12. Manjunath V, Goren J, Fujimoto JG, Duker JS. Analysis of choroidal thickness in age-related macular degeneration using spectral-domain optical coherence tomography. Am J Ophthalmol. 2011;152(4):663-8.

13. Branchini L, Regatieri C, Adhi M, Flores-Moreno I, Manjunath V, Fujimoto JG, et al. Effect of intravitreous anti-vascular endothelial growth factor therapy on choroidal thickness in neovascular age-related macular degeneration using spectral-domain optical coherence tomography. JAMA Ophthalmol. 2013;131(5): 693-4.

14. Kim JH, Kang SW, Kim JR, Kim SJ. Variability of subfoveal choroida thicknessmeasurements in patients with age-related macular degeneration and central serous chorioretinopathy. Eye (Lond). 2013;27(7): 809-15.

15. Hara K. Effects of cigarette smoking on ocular circulation chronic effect on choroidal circulation. Nippon Ganka Gakkai Zasshi. 1991;95: 939-43.

16. Wimpissinger B, Resch $H$, Berisha F, Weigert G, Schmetterer L, Polak K Response of choroidal blood flow to carbogen breathing in smokers and non-smokers. Br J Ophthalmol. 2004;88(6):776-81.
17. Sizmaz S, Küçükerdönmez C, Pinarci EY, Karalezli A, Canan H, Yilmaz G. The effect of smoking on choroidal thickness measured by optical coherence tomography. Br J Ophthalmol. 2013;97:601-4.

18. Ulas F, Celik F, Doğan Ü, Celebi S. Effect of Smoking on Choroidal Thickness in Healthy Smokers. Current Eye Res. 2014:39(5):504-11.

19. Sánchez Ramos JA, Espinosa Soto IC, Ramírez-Estudillo JA. Análisis del grosor coroideo en personas fumadoras obtenido mediante Swept Source-OCT. Rev Mex Oftalmol. 2017;91(6):306-10.

20. Moschos MM, Nitoda E, Laios K, Ladas DS, Chatziralli IP. The Impact of Chronic Tobacco Smoking on Retinal and Choroidal Thickness in Greek Population Oxidative Medicine and Cellular Longevity. 2016;1-7.

21. Tayyab H, Jahangir S, Kishi S. Effect of Chronic Smoking on Choroidal Thickness as Measured by Swept Source OCT When Compared to Non Smokers. Pak J Ophthalmol. 2015;31(4):177-81.

22. Benowitz N, Hukkanen J, Jacob P III. Nicotine Chemistry, Metabolism, Kinetics and Biomarkers. Handb Exp Pharmacol. 2009;(192):29-60.

23. Zengin $\mathrm{MO}$, Cinar $\mathrm{E}$, Kucukerdonmez $\mathrm{C}$. The effect of nicotine on choroidal thickness. Br J Ophthalmol. 2014;98:233-7. 